\title{
Appearance of a fractional Stokes-Einstein relation in water and a structural interpretation of its onset
}

\author{
Limei Xu ${ }^{1,2}$, Francesco Mallamace ${ }^{3 \star}$, Zhenyu Yan ${ }^{2}$, Francis W. Starr ${ }^{4}$, Sergey V. Buldyrev ${ }^{2,5}$ \\ and H. Eugene Stanley ${ }^{2 \star}$
}

The Stokes-Einstein relation has long been regarded as one of the hallmarks of transport in liquids. It predicts that the self-diffusion constant $D$ is proportional to $(\tau / T)^{-1}$, where $\tau$ is the structural relaxation time and $T$ is the temperature. Here, we present experimental data on water confirming that, below a crossover temperature $T_{\times} \approx 290 \mathrm{~K}$, the StokesEinstein relation is replaced by a 'fractional' Stokes-Einstein relation $D \sim(\tau / T)^{-\zeta}$ with $\zeta \approx 3 / 5$ (refs 1-6). We interpret the microscopic origin of this crossover by analysing the $\mathrm{OH}$ stretch region of the Fourier transform infrared spectrum over a temperature range from 350 down to $200 \mathrm{~K}$. Simultaneous with the onset of fractional Stokes-Einstein behaviour, we find that water begins to develop a local structure similar to that of low-density amorphous solid $\mathrm{H}_{2} \mathrm{O}$. These data lead to an interpretation that the fractional Stokes-Einstein relation in water arises from a specific change in the local water structure. Computer simulations of two molecular models further support this interpretation.

We first present our experimental results on water confined in MCM-41-S nanotubes. We measure the self-diffusion $D$ by nuclear magnetic resonance, and we measure the translational relaxation time $\tau$ by using incoherent, quasi-elastic neutron scattering ${ }^{1,2}$ (QENS). Thus, the Stokes-Einstein relation,

$$
D \sim(\tau / T)^{-1}
$$

can be tested. Our data (Fig. 1a) confirm equation (1) at high temperatures, but show that, on cooling below a crossover temperature $T_{\times} \approx 290 \mathrm{~K}$, the Stokes-Einstein relation (1) gives way to a 'fractional Stokes-Einstein relation" ${ }^{2-6}$,

$$
D \sim(\tau / T)^{-\zeta}
$$

with $\zeta \approx 0.62$.

As a first step to obtain a structural interpretation of this fractional Stokes-Einstein behaviour, we turn to measurements of the infrared spectrum ${ }^{1,2,7-9}$. For water, this spectrum can be split into two contributions, one resembling the spectrum of high-density amorphous (HDA) solid $\mathrm{H}_{2} \mathrm{O}$ and the other resembling the spectrum of low-density amorphous (LDA) solid
$\mathrm{H}_{2} \mathrm{O}$. We interpret these two contributions as corresponding to water molecules with more HDA-like local structure, or more LDA-like local structure, respectively ${ }^{10}$. Figure $1 \mathrm{~b}$ shows the relative populations of molecules with locally LDA-like structure and molecules with locally HDA-like structure calculated by decomposition of the infrared spectra. With decreasing $T$, the LDA-like population increases, whereas the HDA-like population decreases. The fractional Stokes-Einstein crossover temperature $T_{\times}$seems to roughly coincide with the onset of the increase of the population of molecules with LDA-like local structure (and a corresponding decrease of the population of the molecules with HDA-like local structure), consistent with the possibility that the changes in intramolecular vibrational properties may be connected to the onset of fractional Stokes-Einstein behaviour.

To more clearly see the change in the relative populations of molecules with LDA-like local structure (and, correspondingly, with HDA-like local structure), we calculate the derivatives of the relative populations with respect to temperature (Fig. 1c). The derivatives of the relative populations become noticeably non-zero at the same value of the crossover temperature, $T_{\times} \approx 290 \mathrm{~K}$. In contrast, we find that the maximal rate of change of the vibrational spectrum occurs at a much lower temperature, $T_{\max } \approx 245 \mathrm{~K}$, approaching the Widom temperature $225 \mathrm{~K}$ for bulk water ${ }^{11}$.

As these experiments examine water confined to cylindrical pores of $\approx 2 \mathrm{~nm}$ diameter, it is natural to question whether the findings might be instructive for understanding bulk water at low $T$. There are two reasons to believe that the answer is yes: (1) computer simulations of confined water on a hydrophilic surface ${ }^{12}$ show that hydrophilic silica-confined water has similar behaviour to bulk water, indicating that the hydrophilic surfaces do not have serious effects on the properties of water, except for significantly lowering the freezing temperature and stabilizing the liquid phase, which enables the study of the supercooled region made impossible in bulk water owing to crystallization; (2) the presence of hysteresis in a temperature cycle (on cooling/heating) is a signature of an interaction between water and silica. However, for the MCM-41-S confined system, only negligible hysteresis was observed by means of X-ray scattering and calorimetric experiments ${ }^{13,14}$. Thus, it is plausible that the MCM-41-S confined water provides information regarding bulk water.

As experiments on bulk water at $T<250 \mathrm{~K}$ are impractical owing to crystallization, we carry out constant- $T$ and constant-density

\footnotetext{
${ }^{1}$ World Premier International (WPI) Research Center, Advanced Institute for Materials Research, Tohoku University, Sendai 980-8577, Japan, ${ }^{2}$ Center for Polymer Studies and Department of Physics, Boston University, Boston, Massachusetts 02215, USA, ${ }^{3}$ Dipartimento di Fisica and CNISM, Universitá di Messina, I-98122, Messina, Italy, ${ }^{4}$ Department of Physics, Wesleyan University, Middletown, Connecticut 06459, USA, ${ }^{5}$ Department of Physics, Yeshiva University, 500 West 185th Street, New York 10033, USA. *e-mail: francesco.mallamace@unime.it; hes@bu.edu.
} 


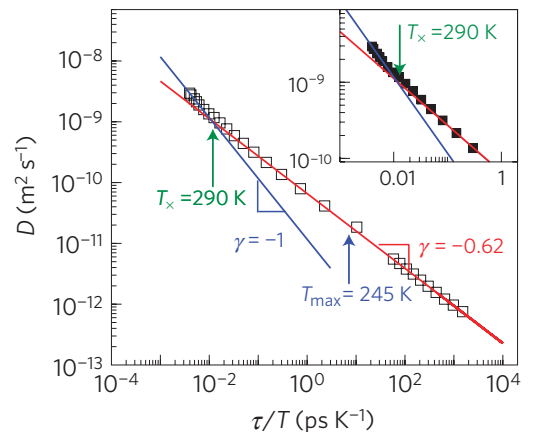

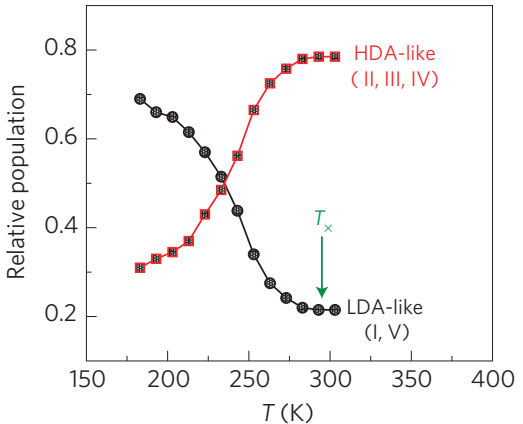

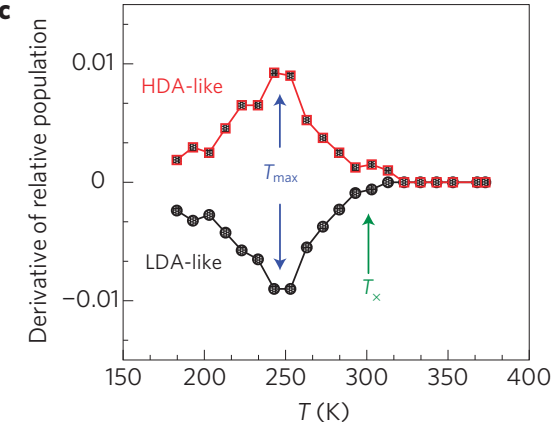

Figure 1 Experimental results for water at $\mathbf{P}=\mathbf{1}$ bar. a, Parametric relation of $D$ as a function of $\tau / T$. The onset of the fractional Stokes-Einstein relation around $T_{X} \approx 290 \mathrm{~K}$ is indicated by the change of slope from $\zeta=1$ to $\zeta=0.62$, whereas $T_{\max } \approx 245 \mathrm{~K}$ is determined from $\mathbf{c}$. $\mathbf{b}$, The relative population of different species of water molecules in experiment. (1) LDA: all molecules represented by group I of the infrared spectra (see the Methods section); (2) HDA: all of the other molecules. c, The derivative of the relative population with respect to temperature for LDA-like and HDA-like species.

a

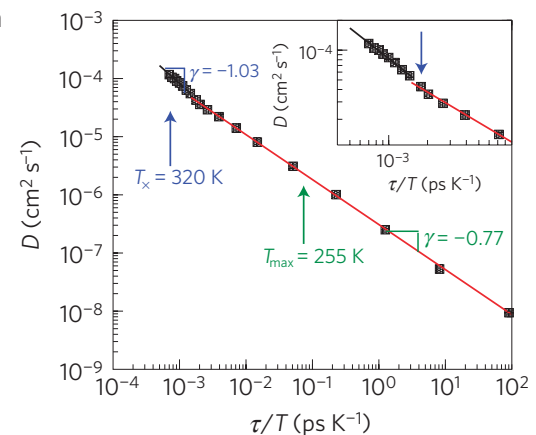

b

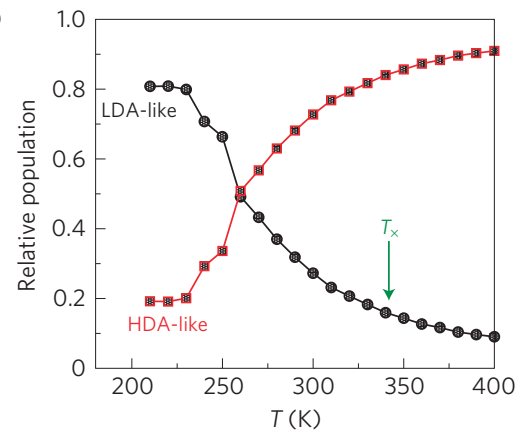

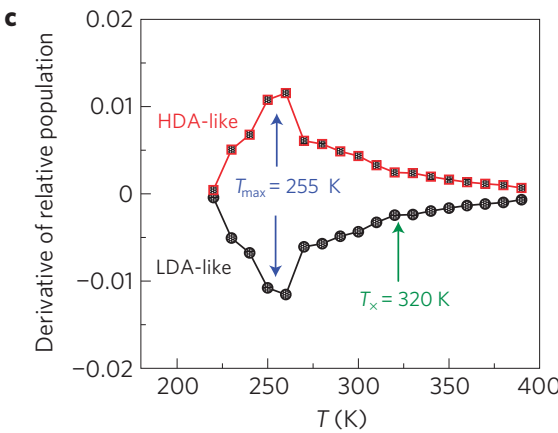

Figure 2 | Analogue of Fig. 1 for bulk TIP5P water. a, Parametric relation of $D$ as a function of $\tau / T$. The Stokes-Einstein relation breaks down around $T \approx 320 \mathrm{~K}$, whereas $T_{\max } \approx 255 \mathrm{~K}$. b. The relative population of different species of molecules in the simulation for TIP5P along paths of constant density $\rho=1 \mathrm{~g} \mathrm{~cm}^{-3}$. The crossing of relative populations of LDA and HDA corresponds to the temperature where the specific heat $C_{P}$ shows a maximum, similar to the experimental results in Fig. 1b. c, Derivative of the relative population with respect to temperature. For each species (LDA and HDA), the maximum change occurs at $T_{\max } \approx 255 \mathrm{~K}$. The breakdown of the Stokes-Einstein relation occurs at temperature $T_{\times}$when each species starts to change more rapidly.

molecular dynamics simulations of $N=512$ water molecules interacting with the TIP5P potential ${ }^{15}$ at a fixed density $\rho=1 \mathrm{~g} \mathrm{~cm}^{-3}$. In addition, direct access to the molecular coordinates makes it possible to connect the changes in $D$ to changes in the local molecular structure.

The relaxation time $\tau$ is defined as the time when the coherent intermediate scattering function decays by a factor of $e$ for the wave vector $\mathbf{q}$ of the first peak of the static structure factor. The diffusion coefficient is computed from the root-mean-square displacement of the oxygens as a function of temperature. Analogous to the experimental results in Fig. 1a, we show the simulation results of TIP5P water for $D$ as a function of $\tau / T$ (Fig. 2a). We see that below $T_{\times} \approx 320 \mathrm{~K}$ the Stokes-Einstein relation crosses over to a fractional Stokes-Einstein relation ${ }^{16}$ of equation (2) with $\zeta=0.77$.

We next use our simulations to make a connection to local structure, rather than intramolecular vibration. This provides a more intuitive connection to the real space structure of the fluid. Similar to the experimental approach, we wish to relate the onset of the fractional Stokes-Einstein relation to the emergence of LDA-like local structure in the liquid. We identify different local structures by carrying out a direct calculation for each molecule $i=1,2, \ldots, N$ of the local tetrahedral structural order parameter $Q_{i}$ (ref. 17), defined as

$$
Q_{i} \equiv 1-\frac{3}{8} \sum_{j=1}^{3} \sum_{k=j+1}^{4}\left(\cos \phi_{j k}+\frac{1}{3}\right)^{2}
$$

where $\phi_{j k}$ is the angle formed by the lines joining the oxygen atom of molecule $i$ with pairs of its four nearest neighbours $j$ and $k$.
The possible values of $Q_{i}$ vary between $Q_{i}=0$ for the limit of uncorrelated angles and $Q_{i}=1$ for the perfect tetrahedral network.

For each molecule, we calculate its local orientational order $Q_{i}$. We assign a locally 'LDA-like' molecule if $Q_{i}>0.8$, and an 'HDAlike' molecule if $Q_{i} \leq 0.8$. Our decomposition to different structural groups is based on the probability density function $P(Q)$. As shown in Fig. $2 \mathrm{~d}$ of ref. 18, for density $\rho=1 \mathrm{~g} \mathrm{~cm}^{-3}, P(Q)$ changes with temperature. At very low temperature $(T=240 \mathrm{~K}), P(Q)$ has one dominant peak near $Q \approx 0.9$, indicating a more tetrahedral local structure similar to ice. At intermediate temperatures, $P(Q)$ starts to develop a shoulder at $Q \approx 0.5$, indicating a change in the population of the local structure; and at higher temperatures $(T=340 \mathrm{~K}), P(Q)$ has a broader distribution with two peaks at $Q \approx 0.5$ and $Q \approx 0.9$, respectively. Thus, the decomposition of the local structure to (1) the low-density-liquid-like structure (locally tetrahedral structure in the first shell with $Q \approx 0.9)$ and $(2)$ the high-density-liquid-like structure (non-tetrahedral structure with $Q<0.8)$ is a reasonable and valid decomposition based on the number of hydrogen bonds and the distribution of the local tetrahedral order parameter $Q$. We can count $n_{\mathrm{L}}$ and $n_{\mathrm{H}}$ the number of molecules with 'LDA-like' and 'HDA-like' local structures, respectively. The relative populations are defined as $n_{\mathrm{L}} / N$ and $n_{\mathrm{H}} / N$, as shown in Fig. $2 \mathrm{~b}$.

We observe in Fig. 2b a gradual increase in LDA-like local structures, and a decrease in HDA-like local structure. The derivatives of the relative populations of the LDA-like and the HDA-like molecules with respect to temperature (Fig. 2c) show that the change does not have a sharp onset at $T_{\times} \approx 320 \mathrm{~K}$. For each species (LDA-like and HDA-like), the maximum change defines 

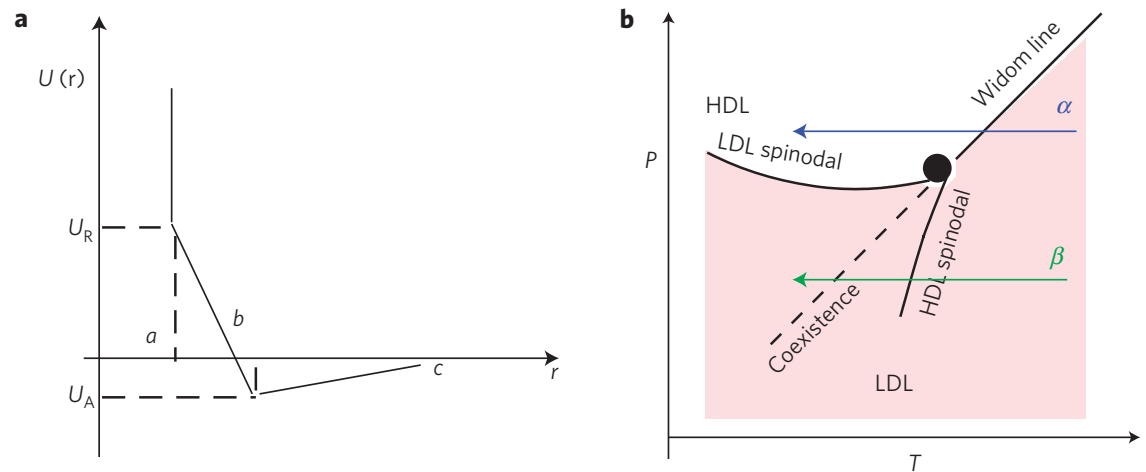

Figure 3 | Definition and properties of the Jagla model potential. a, The spherically symmetric 'two-scale' Jagla ramp potential with attractive and repulsive ramps. Here, $U_{R}=3.56 U_{0}, U_{A}=-U_{0}, a$ is the hard-core diameter, $b=1.72 a$ is the soft-core diameter and $c=3 a$ is the long-distance cutoff. b, Sketch of the phase diagram for the Jagla potential. Studies along path $\alpha$ in the one-phase region are reported in this letter. Unlike water, the HDA phase in the ramp model is a more ordered structure (lower entropy $S$ ) than the LDA phase (higher entropy), leading to a positively sloped coexistence line because $\Delta S / \Delta V>0$. LDL: low-density liquid; HDL: high-density liquid.
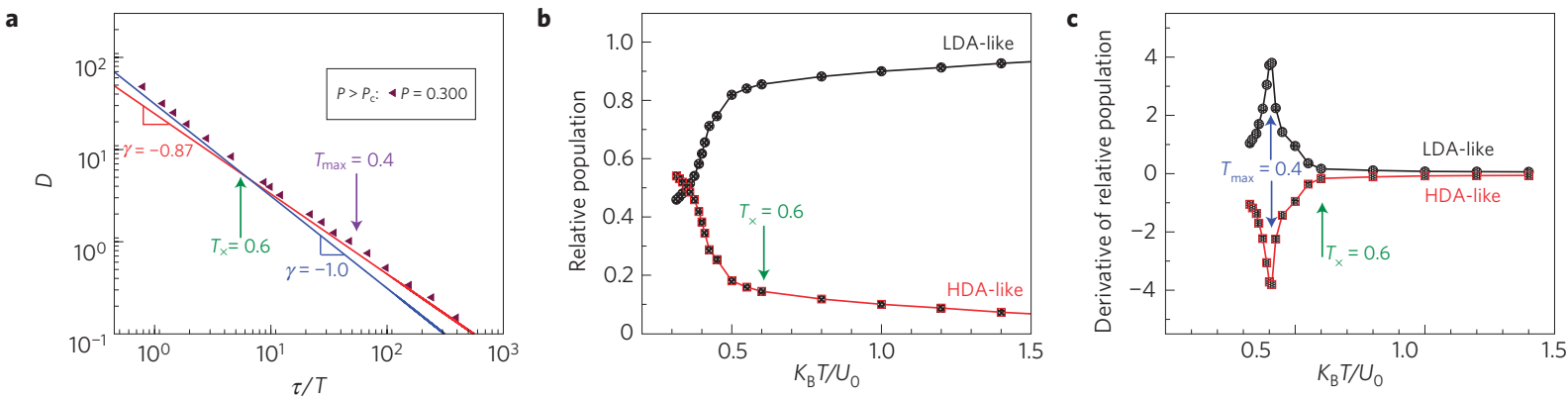

Figure 4 | Analogue of Fig. 1 for the two-scale spherically symmetric Jagla model. a, The Jagla system obeys the fractional Stokes-Einstein relation with $\zeta \approx 0.87$ for different paths over a broad range of temperatures, and follows the normal Stokes-Einstein relation with $\zeta=1$ at high temperatures. The breakdown occurs roughly around $T_{\times} \approx 0.6$. b. Relative population of HDA-like and LDA-like molecules in the Jagla liquid along the constant-pressure path $P=0.3$. c, The derivatives of relative populations with respect to temperature. Similar to the results for TIP5P shown in Fig. $2 c$, the maximal changes occur at $T_{\max } \approx 0.40$, and the breakdown occurs at temperature $T_{\times} \approx 0.60$ where the relative population starts to show rapid changes.

a temperature $T_{\max } \approx 255 \mathrm{~K}$ for the structural evolution ${ }^{19,20}$. Like our experimental results, $T_{\times}>T_{\max }$ for TIP5P, indicating that the change to fractional Stokes-Einstein behaviour can be connected with the emergence of more highly structured regions of the liquid, rather than the maximal rate of change.

To explore whether this behaviour is specific to water, or if similar behaviour occurs for other pure substances that may possibly possess a liquid-liquid critical point ${ }^{21,22}$, we also study the two-scale spherically symmetric Jagla ramp model ${ }^{23,24}$ of a liquid (Fig. 3). This model reproduces the thermodynamic and dynamical anomalies of liquid water, as well as exhibiting a liquid-liquid critical point. For the Jagla potential (Fig. 3 ) $^{23}$, we implement a discrete molecular dynamics simulation for $N=1,728$ particles interacting through step potentials in a constant-temperature and constant-volume ensemble.

We examine the Stokes-Einstein relation and relative populations along a constant-pressure path $P=0.30$ that remains in the one-phase region (path $\alpha$ of Fig. 3 ) $^{23}$. Figure 4 shows for the Jagla model the analogue of Figs 1 and 2. We find for $T>T_{\times}$the normal Stokes-Einstein relation with $\zeta=1$, and for $T<T_{\times} \approx 0.6$ a fractional Stokes-Einstein relation with $\zeta \approx 0.87$.

Analogous to the structural analysis done for TIP5P, we next consider the relative population of HDA-like and LDA-like molecules in the Jagla liquid. In the Jagla liquid, the changes in the structure can be deduced from the number of particles in the first and second coordination shell. In our study, for each molecule, we calculate its number of nearest neighbours, $n$, within a distance $r / a \leq 1.3$ (first minimum in pair correlation function).
We define a locally 'LDA-like' molecule if $n<2$, and an 'HDA-like' molecule if $n \geq 2$. Thus, we can calculate the relative populations $n_{\mathrm{L}} / N$ and $n_{\mathrm{H}} / N$. Figure $4 \mathrm{~b}$ shows that the relative population of the more ordered phase (HDA-like for the Jagla model and LDA-like for water) starts to increase sharply at $T_{\times} \approx 0.6$, whereas the relative population of the less-ordered phase (LDA for the Jagla model) starts to decrease, just as observed experimentally and in the TIP5P model.

Similar to the experimental and TIP5P results, the temperature derivative of relative populations (Fig. 4c) shows that the maximum change occurs at a temperature $T_{\max } \approx 0.4$ below the onset temperature of the sharp changes of the relative populations, that is, $T_{\max }<T_{\times}$. The maxima we observe in the rates of change of the different species of molecules is reminiscent of the observation that thermodynamic response functions exhibit maxima in the supercooled region of water. The constant-pressure specific heat is the most commonly examined of these response functions, and the locus of the specific-heat maxima is often referred to as the Widom line ${ }^{19}$. Hence, it is natural to expect that there may be some connection between the location of the maximal rates of change in the molecular structure we measure and the Widom temperature for that pressure ${ }^{25}$.

For the Jagla model, $T_{\max }$ coincides closely with the Widom temperature $T_{\mathrm{W}}(P)$. The Jagla model has a liquid-liquid critical point at $T_{\mathrm{c}}=0.375, P_{c}=0.245$ (ref. 24), and we consider cooling along the $P=0.3$ isobar, near to the critical pressure. As response functions must diverge at the critical point, the locus of any maxima must become asymptotically close as we approach the critical point. 
Therefore, the coincidence between $T_{\mathrm{W}}$ and $T_{\max }$ is expected. As a result, the breakdown of the Stokes-Einstein relation is influenced by the liquid-liquid critical point.

For the experimental results and TIP5P water, we work at a pressure that is much lower than that of the expected critical pressure for each system. As we work far away from the critical point, the positions of the temperature maxima of the various response functions may differ by a significant amount, as they must coincide only near the critical point ${ }^{26}$. For TIP5P, we find that $T_{\mathrm{W}} \approx 255 \mathrm{~K}$, identical to $T_{\max }$. This suggests that the loci for the maximum in specific heat and the maximal rate of change of the local structure coincide even at relatively low pressure. However, for water, we found a larger difference, with $T_{\mathrm{W}} \approx 225 \mathrm{~K}$ (ref. 11) and $T_{\max } \approx 245 \mathrm{~K}$.

Both our experimental findings and our simulation results are consistent with the possibility that, in water, the fractional Stokes-Einstein relation (2) sets in near the temperature where the relative populations of molecules with LDA-like and HDAlike local structures start to rapidly change. A structural origin for the failure of the Stokes-Einstein relation can be understood by recognizing that the Stokes-Einstein relation defines an effective hydrodynamic radius. The different species have different hydrodynamic radii, so when their relative populations change, the classical Stokes-Einstein relation (based on the assumption of the fixed hydrodynamic radius) breaks down. Moreover, a connection between the local structure of water and its dynamics is expected ${ }^{16,27,28}$; molecules with a locally tetrahedral geometry are more 'sluggish' than less well-networked molecules. This effect also occurs in solutions, where a failure of the scaling between diffusion and relaxation has been interpreted in terms of changes in the local network structure ${ }^{29}$. For these reasons, our structure-based interpretation for the failure of the Stokes-Einstein relation is particular to water, and the breakdown occurs at almost twice the glass-transition temperature $T_{\mathrm{g}}$. An explanation for the failure of the Stokes-Einstein relation for liquids in general, where the breakdown typically occurs within $20-40 \%$ of $T_{\mathrm{g}}$, must involve understanding how the intermittency of the molecular motion couples to diffusion and relaxation mechanisms approaching the glass transition. Such a general explanation should also be applicable to water, where the emergence of intermittency of the dynamics occurs at unusually high $T$ owing to water's unusual thermodynamics and corresponding changes in the fluid structure.

\section{Methods}

We carried out infrared absorption measurements at ambient pressure in the oxygen-hydrogen stretching (OHS) vibrational spectral regions. We used Bomem DA8 Fourier transform spectrometer, operating with a Globar source, in combination with a $\mathrm{KBr}$ beam splitter and a DTGS/KBr detector. To avoid saturation effects, we used the attenuated total reflection geometry, which is generally insensitive to sample thickness. The spectra of interest were recorded at a resolution of $4 \mathrm{~cm}^{-1}$, automatically adding 200 repetitive scans to obtain a good signal-to-noise ratio and highly reproducible spectra. They were then appropriately normalized by taking into account the effective number of absorbers. Our spectra for confined water were not smoothed, and the only manipulation used was the baseline adjustment. Investigated samples (water confined in MM-41-S annotates) have hydration levels of $H \sim 0.5$ ( $0.5 \mathrm{~g}$ of $\mathrm{H}_{2} \mathrm{O}$ per gram of MM-41-5).

Different approaches, such as mixture and continuous models developed in terms of scattering theory, have been applied to analyse the spectra of water by means of Gaussian components unambiguously classified as hydrogen-bonded or non-hydrogen-bonded OHS oscillators by considering different hydrogen-bonded geometries $^{30-32}$. We work in an intermediate picture between the continuous and discrete models, assuming the existence of a percolating hydrogen-bond network with a characteristic hydrogen-bond lifetime of the order of a picosecond. The Gaussian components' peaks are located at: (I) $3,120 \mathrm{~cm}^{-1}$, (II) $3,220 \mathrm{~cm}^{-1}$, (III) $3,500 \mathrm{~cm}^{-1}$ and (IV) $3,620 \mathrm{~cm}^{-1}$.

The network contributes to the OHS vibrational spectra with two main collective modes. The first, at $3,120 \mathrm{~cm}^{-1}$, is assigned to fully bonded water molecules, owing to its similarity to the OHS band observed in low-density amorphous LDA ice ${ }^{30}$. The second, at $3,220 \mathrm{~cm}^{-1}$, is associated with water molecules having an average degree of connectivity larger than that of dimers, but lower than those in a hydrogen-bonded tetrahedral structure. The highest wavenumber components are the only ones present in bulk water in the temperature region $600<T<640 \mathrm{~K}$ near the liquid-gas critical point $T_{\mathrm{c}}=647 \mathrm{~K}$, and so they are ascribed to dimeric and monomeric water.

In summary, the OHS spectra of water can be decomposed into three main groups: (I), (II and III) and (IV), where (II-III and IV) represent the HDA-like water. Components I and II make the largest contribution to the OHS spectra in the supercooled region. Using this classification, the relative population of local structures in water can be calculated on the basis of the decomposition, which is defined as the relative area (the ratio of each component's integrated area to the total OHS integrated area $)^{1}$. The overall continuity we found between supercooled and amorphous water (see, for example, Fig. 3 of ref. 1) in the infrared spectral parameter supports the experimental validity of our data interpretation.

For the microscopic average correlation time, our experiment used incoherent QENS and the spectral data were analysed by using the relaxing cage model. The typical wave vector is in the range $0.2 \AA^{-1}<\mathrm{q}<2 \AA^{-1}$. A proper choice of the energy resolution and of the dynamical range is also important for this type of QENS experiment. We use two spectrometers, one with an energy resolution of $0.8 \mu \mathrm{V}$ and a dynamical range of $\pm 11 \mu \mathrm{V}$ and the other with an energy resolution of $20 \mu \mathrm{V}$ and a dynamical range of $\pm 0.5 \mathrm{mV}$. Further experimental details are given in refs 1,2 .

Received 26 June 2008; accepted 29 May 2009; published online 5 July 2009

\section{References}

1. Mallamace, F. et al. Evidence of the existence of the low-density liquid phase in supercooled, confined water. Proc. Natl Acad. Sci. USA 104, 424-428 (2007).

2. Chen, S.-H. et al. The violation of the Stokes-Einstein relation in supercooled water. Proc. Natl Acad. Sci. USA 103, 12974-12978 (2006).

3. Becker, S. R., Poole, P. H. \& Starr, F. W. Fractional Stokes-Einstein and Debye-Stokes-Einstein relations in a network-forming liquid. Phys. Rev. Lett. 97, 055901 (2006)

4. Mazza, M. G., Giovambattista, N., Stanley, H. E. \& Starr, F. W. A breakdown of the Stokes-Einstein and Stokes-Einstein-Debye relations and dynamics heterogeneities in a molecular system. Phys. Rev. E 76, 031203 (2007).

5. Fernandez-Alonson, F. et al. Observation of fractional Stokes-Einstein behavior in the simplest hydrogen-bonded liquid. Phys. Rev. Lett. 98, 077801 (2007).

6. Douglas, J. F. \& Leporini, D. Obstruction model of the fractional Stokes-Einstein relation in glass-forming liquids. J. Non-Cryst. Solids 235, 137-141 (1998).

7. Soper, A. K. \& Ricci, M. A. Structures of high-density and low-density water. Phys. Rev. Lett. 84, 2881-2884 (2000).

8. Zanotti, J. M., Bellissent-Funel, M. C. \& Chen, S.-H. Experimental evidence of a liquid-liquid transition in interfacial water. Europhys. Lett. 71, 91-97 (2005).

9. Walrafen, G. E., Hokmababi, M. S. \& Yang, W. H. A. Raman isosbestic points from liquid water. J. Chem. Phys. 85, 6964-6969 (1986).

10. Canpolat, M. et al. Local structural heterogeneities in liquid water under pressure. Chem. Phys. Lett. 294, 9-12 (1998).

11. Starr, F. W., Angell, C. A. \& Stanley, H. E. Entropy and dynamic properties of water below the homogeneous nucleation temperature. Physica A 323, 51-66 (2003).

12. Giovambattista, N., Rossky, P. J. \& Debenedetti, P. G. Effect of pressure on the phase behavior and structure of water confined between nanoscale hydrophobic and hydrophilic plates. Phys. Rev. E 73, 041604 (2006).

13. Morishige, K. \& Nobuoka, K. X-ray diffraction studies of freezing and melting of water confined in a mesoporous adsorbent (MCM-41). J. Chem. Phys. 107, 6965-6969 (1997).

14. Schreiber, A., Ketelsen, I. \& Findenegg, G. H. Melting and freezing of water in ordered mesoporous silica materials. Phys. Chem. Chem. Phys. 3 , 1185-1195 (2001).

15. Yamada, M., Mossa, S., Stanley, H. E. \& Sciortino, F. Interplay between time-temperature-transformation and the liquid-liquid phase transition in water. Phys. Rev. Lett. 88, 195701 (2002).

16. Mazza, M. G., Giovambattista, N., Starr, F. W. \& Stanley, H. E. Relation between rotational and translational dynamic heterogeneities in water. Phys. Rev. Lett. 96, 057803 (2006).

17. Errington, J. R. \& Debenedetti, P. G. Relationship between structural order and the anomalies of liquid water. Nature 409, 318-321 (2001).

18. Yan, Z. et al. Structure of the first- and second-neighbour shells of simulated water: Quantitative relation to translational and orientational order. Phys. Rev. E 76, 051201 (2007).

19. $\mathrm{Xu}, \mathrm{L}$. et al. Relation between the Widom line and the dynamic crossover in systems with a liquid-liquid phase transition. Proc. Natl Acad. Sci. USA 102, 16558-16562 (2005).

20. Kumar, P. et al. Relation between the Widom line and the breakdown of the Stokes-Einstein relation in supercooled water. Proc. Natl Acad. Sci. USA 104 9575-9579 (2007)

21. Sheng, H. W. et al. Polyamorphism in a metallic glass. Nature Mater. 6, 192-197 (2007)

22. Hsu, C. W., Largo, J., Sciortino, F. \& Starr, F. W. Hierarchies of networked phases induced by multiple liquid-liquid critical points. Proc. Natl Acad. Sci. USA 105, 13711-13715 (2008). 
23. Xu, L., Buldyrev, S. V., Angell, C. A. \& Stanley, H. E. Thermodynamics and dynamics of the two-scale spherically symmetric Jagla ramp model of anomalous liquids. Phys. Rev. E 74, 031108 (2006).

24. Xu, L., Buldyrev, S. V., Giovambattista, N., Angell, C. A. \& Stanley, H. E. A monatomic system with a liquid-liquid critical point and two distinct glassy states. J. Chem. Phys. 130, 054505 (2009).

25. Moore, E. B. \& Molinero, V. Growing correlation length in supercooled water. J. Chem. Phys. (in the press).

26. Poole, P. H., Saika-Voivod, I. \& Sciortino, F. Density minimum and liquid-liquid phase transition. J. Phys. Condens. Matter 17, L431-L437 (2005).

27. Sciortino, F., Geiger, A. \& Stanley, H. E. Effect of defects on molecular mobility in liquid water. Nature 354, 218-221 (1991).

28. Giovambattista, N., Starr, F. W., Sciortino, S., Buldyrev, S. V. \& Stanley, H. E. Transitions between inherent structures in water. Phys. Rev. E 65, 041502 (2002).

29. Molinero, V., Cağin, T. \& Goddard, W. A. Mechanisms of nonexponential relaxation in supercooled glucose solutions: The role of water facilitation. J. Phys. Chem. A 108, 3699-3712 (2004).
30. Walrafen, G. E. in Structure of Water and Aqueous Solutions (ed. Luck, W. A. P.) 248-284 (Verlag Chemie, 1974).

31. Auer, B. M. \& Skinner, J. L. IR and Raman spectra of liquid water: Theory and interpretation. J. Chem. Phys. 128, 224511 (2008).

32. Kumar, R., Schmidt, J. R. \& Skinner, J. L. Hydrogen bonding definitions and dynamics in liquid water. J. Chem. Phys. 126, 204107 (2007).

\section{Acknowledgements}

We thank C. A. Angell, P. G. Debenedetti and V. Molinero for helpful discussions, and the NSF Chemistry Program for support. X.L.M. acknowledges the support by the World Premier International Research Center Initiative (WPI Initiative), MEXT, Japan. F.W.S acknowledges NSF grant DMR-0427239. S.V.B. acknowledges partial support through the Bernard W. Gamson Computational Science Center at Yeshiva College.

\section{Additional information}

Reprints and permissions information is available online at http://npg.nature.com/ reprintsandpermissions. Correspondence and requests for materials should be addressed to F.M. or H.E.S. 